\title{
Importância dos enfermeiros na identificação do Cyberbullying: Revisão sistemática
}

\section{Importance of nurses in the identification of cyberbullying: Systematic review}

\author{
José Mendes (1) \\ Susana Queirós (2) \\ Marina Pedro (2) \\ Marta Oliveira (3) \\ (1) INTELECTO - Psicologia \& Investigação, Ponta Delgada, Açores; Unidade de Saúde de São Miguel, Centro de Saúde \\ do Nordeste, Açores, Portugal \\ (2) Unidade de Saúde da Ilha de São Miguel, Centro de Saúde da Ribeira Grande, Açores, Portugal \\ (3) Unidade de Cuidados de Saúde Personalizados de Vendas Novas, Centro de Saúde de Vendas Novas, Vendas Novas, \\ Portugal
}

Recebido: 18/12/2018; Revisto: 19/01/2019; Aceite: 19/02/2019.

https://doi.org/10.31211/rpics.2019.5.1.105

\section{Resumo}

Objetivo: O cyberbullyingtem despertado a atenção na comunidade científica, existindo já uma maior preocupação por parte dos órgãos políticos perante um tema que se revela uma preocupação de saúde pública. Com a presente revisão sistemática da literatura pretende-se compreender a abordagem dos cuidados de saúde primários na identificação e prevenção do cyberbullying em crianças/jovens. Métodos: Com recurso a várias bases de dados (PubMed, Google Scholar, Web of Science e EBSCO), pesquisaram-se artigos científicos utilizando os operadores booleanos AND e NOT com as palavras chave Cyberbullying, Child, Adolescent e Primary Health Care. Incluíram-se os artigos entre os anos 2013 e 2018 com resumo e texto completo. Resultados: Identificaram-se no total sessenta e três artigos. Vinte e três artigos eram duplicados, onze artigos eram periódicos de revistas consideradas de cariz não-científico. Após leitura integral, eliminaram-se vinte e quatro artigos, em que somente cinco cumpriram com os critérios de inclusão. Conclusão: As Tecnologias de Informação e Comunicação apresentam benefícios e malefícios, onde a família/pessoa significativa e os enfermeiros desempenham um papel primordial na prevenção e antecipação de comportamentos de risco para o desenvolvimento integral da criança/jovem. Justifica-se um maior investimento na formação de profissionais de saúde, dotando-os de estratégias de avaliação e intervenção na prevenção do cyberbullying, identificando vítimas e agressores em todos os contextos (escolar, familiar, cuidados de saúde primário e emergência hospitalar). Não tendo sido encontrados estudos realizados na Europa, sugere-se maior investigação que permita melhor compreender a promoção positiva do cuidar da criança/jovem e família perante o cyberbullying.

Palavras-Chave: Cyberbullying; Crianças; Adolescente; Enfermeiros; Cuidados de Saúde Primários.

\section{DI\&D | ISMT}

rpics@ismt.pt

https://rpics.ismt.pt
Publicação em Acesso Aberto

(02019. O(s) Autor(es). Este é um artigo de acesso aberto distribuído sob a Licença Creative Commons Attribution, que permite uso, distribuição e reprodução sem restrições em qualquer meio, desde que o trabalho original seja devidamente citado.
José Mendes

INTELECTO - Psicologia e Investigação Rua do Monte, 52B, Fração L, R/C Esq.

9500-451 Ponta Delgada

Açores, Portugal,

e-mail: josemendes@intelecto.pt 


\begin{abstract}
Objective: Cyberbullying has been drawing the attention of the scientific community. Therefore, nowadays, there is a greater concern on the part of political groups towards this subject which is a public health matter. This systemic literature review aims to understand the approach of primary health care in the identification and prevention of cyberbullying in children/youth. Methods: Following descriptors Cyberbullying, Child, Adolescent, Nurse, and Primary Health Care with Boolean operator AND/NOT in the databases (PubMed, Google Scholar, Web of Science, and EBSCO). The articles between 2013 and 2018 were taken into account, with the summary and full text. Results: Sixty-three articles were identified. Twentythree articles were duplicates, and eleven articles were periodicals of scientific journals. After reading comprehensively, twenty-four articles were eliminated, and only five met the inclusion criteria. Conclusions: Information and Communication Technologies have benefits and disadvantages, with the significant family/person and the nurses play a primary role in the prevention and anticipation of risk behaviors for the overall development of the child/youth. The present Systemic Literature Review alerts for a greater investment in the training of health professionals, providing them with evaluation and intervention strategies to prevent Cyberbullying, identifying victims and aggressors in all contexts (school, family, primary health care, and emergency). Since no studies were found in Europe, more research is suggested to better understand the positive promotion of child/youth care and the family facing Cyberbullying.
\end{abstract}

Keywords: Cyberbullying; Child; Adolescent; Nurses; Primary Health Care.

\title{
Introdução
}

Na última década, o cyberbullying tem sido alvo de maior atenção por parte de investigadores de várias áreas do saber. A utilização da internet apresenta quer oportunidades de aprendizagem, participação cívica, criatividade e ligações sociais, quer riscos que podem ser comerciais, sexuais, julgamento de valores e comportamentos agressivos (Simões, 2010). O plano estratégico do Conselho da Europa sobre os Direitos da Criança defende os direitos da criança no ambiente digital, onde a participação e proteção das crianças deve ser mediada pela criação e disseminação de aplicações que possam capacitar as crianças, pais e educadores a utilizar as novas tecnologias de comunicação e informação de modo seguro (Conselho da Europa, 2016).

Apesar desta preocupação global, não podemos esquecer a intervenção do enfermeiro na prestação de cuidados primários, uma vez que as consequências do cyberbullying podem influenciar o estado de saúde da criança/adolescente e prejudicar o seu adequado desenvolvimento (Caetano et al., 2017). A fase inicial da adolescência é marcada pela mudança da aparência, onde o adolescente pode ajustar o seu comportamento para se enquadrar nas normas dos seus pares, podendo ser vítimas ou participar de bullying (por exemplo, assédio moral), sentindo-se confusos relativamente à sua identidade sexual (United Nations Children's Fund, 2011).

O bullying envolve agressões intencionais, repetitivas e praticadas em uma relação de desigualdade de poder entre vítimas e agressores (Silva et al., 2018), existindo quatro características que distinguem o cyberbullying do bullying tradicional: o anonimato do agressor; o ataque poder acontecer a qualquer momento, independentemente da proximidade física; a interação entre o agressor e vítima é menos pessoal, agravando o comportamento malicioso e a natureza generalizada do cyberbullying como mais penetrante (Carpenter \& Hubbard, 2014). 
O comportamento dos adolescentes perante as novas Tecnologias da Informação e da Comunicação (TIC) têm despertado a atenção de investigadores na última década em Portugal (Amado, Matos, Pessoa, \& Jager, 2009), uma vez que o bullying passou a manifestar-se através de um espaço virtual, denominandose de cyberbullying (Associação Portuguesa de Apoio à Vítima [APAV], 2011; João, João, \& Portelada, 2011; Pigozi \& Machado, 2015). O cyberbullying pode ser definido pelo envio ou post de textos ou imagens que podem ser prejudiciais ou violentos através da internet ou outros dispositivos de TIC (Pessoa \& Amado, 2014).

O comportamento do cyberbullying efetiva-se pela disseminação de informação negativa/falsa com intenção de difamar, importunar e injuriar a vítima (APAV, 2011), existindo uma forte ligação dos jovens relativamente ao telemóvel e ao Messenger (Amado et al., 2009), em que as consequências podem ser devastadoras na saúde física e mental dos adolescentes tais como: dificuldades de concentração, pesadelos, insónias, irritabilidade, cansaço, sentimentos de insegurança, perda de equilíbrio, medo, ansiedade e crises de choro (João et al., 2011).

O fácil acesso à internet, dispositivos móveis e redes sociais pelos adolescentes tem aumentado a exposição ao bullying virtual, tendo o cyberbullying crescido como uma preocupação de saúde pública, podendo afetar a saúde mental dos jovens (Betz, 2011; Carpenter \& Hubbard, 2014). A abordagem multidisciplinar no âmbito dos cuidados de saúde (médicos, psicólogos, assistentes sociais e enfermeiros) é fundamental na promoção e educação para a saúde (Moreno \& Vaillancourt, 2017).

Salienta-se que atualmente as interações online têm impacto nas amizades e nas relações entre os pares, onde os esforços para prevenir o bullying virtual devem concentrar-se no uso que os adolescentes fazem da internet. O risco do cyberbullying está associado a múltiplos fatores e à possibilidade de as relações que se geram entre eles contribuírem para o agravamento do problema (Moreno \& Vaillancourt, 2017). É importante que todos estejam informados e que se discuta esta temática, onde a rede de suporte assume um duplo significado (ter uma rede física e virtual) em cooperação (Almeida \& Gouveia, 2016). Tendo em vista a obtenção contínua de ganhos em saúde para a população adolescente, é fundamental o desenvolvimento de estratégias de intervenção adequadas às problemáticas atuais. De acordo com o Programa Nacional de Saúde Infantil e Juvenil, o cyberbullying surge como um problema social que não deve ser desvalorizado pelos profissionais de saúde que realizam as consultas de vigilância de saúde juvenil. Em contexto de cuidados de saúde primários, torna-se fundamental a abordagem desta temática no âmbito preventivo, com o desenvolvimento de estratégias que devem incluir programas de educação para a saúde, promovendo o desenvolvimento pessoal e social e a autodeterminação dos jovens. Os enfermeiros devem abordar os jovens com o intuito de promover a responsabilização pelas escolhas relativas à saúde, prevenindo situações disruptivas ou de risco acrescido. No âmbito da deteção precoce, torna-se fundamental identificar, apoiar e orientar os jovens e famílias vítimas de maus-tratos e de violência (Direção-Geral de Saúde, 2013).

Sendo o cyberbullying um problema de saúde pública (Direção-Geral de Saúde [DGS], 2015), devem os enfermeiros promover práticas de cuidados que respeitem os direitos humanos; conceber, gerir e colaborar em programas de melhoria nos cuidados de saúde, otimizando a resposta multidisciplinar (Regulamento n. ${ }^{\circ}$ 140/2019, 2019); cabe ainda aos enfermeiros especialistas em enfermagem de saúde da criança e do jovem que possuem competência específica e fundamental, assistir e cuidar da 
criança/jovem e família em situações de especial complexidade, na maximização da sua saúde (Regulamento n. ${ }^{\circ}$ 123/2011, 2011).

Os Padrões de Qualidade, que regulamentam a atividade, reiteram que é da competência do Enfermeiro Especialista em Enfermagem de Saúde da Criança e do Jovem focalizar a sua intervenção na interdependência criança/jovem/família e ambiente, considerando os fatores protetores e de risco associados à sua vivência, ajudando a criança/jovem a alcançar o máximo potencial de saúde e prevenindo complicações para a sua saúde, através da identificação de evidências fisiológicas e emocionais de mal-estar psíquico, bem como a identificação de situações de risco para a criança e jovem (Regulamento n. ${ }^{\circ}$ 351/2015, 2015).

Desta forma, o presente estudo pretende realizar uma revisão sistemática da literatura, com o objetivo de compreender a abordagem dos cuidados saúde primários perante o adolescente, vítima/agressor de cyberbullying na promoção do seu bem-estar biopsicossocial. Assim, pretende-se compreender qual a abordagem dos profissionais de saúde nos cuidados de saúde primários perante a criança/adolescente, vítima/agressor de cyberbullying.

\section{Método}

Tomando em consideração a metodologia de pesquisa a realizar para a presente revisão sistemática da literatura, procederam-se às seguintes etapas: definição da questão de investigação; elaboração dos critérios de elegibilidade; realização de uma pesquisa nas bases de dados; examinação dos artigos a considerar elegíveis; apreciação da qualidade metodológica dos estudos; descrição dos resultados e conclusões e elaboração de uma tabela síntese das características dos estudos.

Procedeu-se à pesquisa das palavras Cyberbullying, Adolescent, Nursee Primary Health Carenas bases de dados Pubmed, Google Scholar, Web of Science e Ebsco. Utilizando os operadores booleanos AND e NOT, foram elaboradas cinco equações de pesquisa: [Cyberbullying AND Adolescent NOT Child/Adult], [Cyberbullying AND Adolescent AND Primary Health Care NOT Child/Adult], [Cyberbullying AND Nurse AND Primary Health Care NOT Child/Adult], [Cyberbullying AND Adolescent AND Nurse NOT Child/Adult] e [Cyberbullying AND Adolescent AND Nurse AND Primary Health Care NOT Child/Adult]. Estas cinco equações foram pesquisadas no texto integral.

Os critérios de inclusão adotados foram: 1) a publicação possuir uma relação entre o cyberbullying, profissionais de saúde, os adolescentes e os cuidados de saúde primários; 2) ser uma publicação na língua portuguesa, inglesa, francesa ou espanhola e 3) ser uma publicação em revistas de cariz científico em texto completo entre o ano 2013 e o ano 2018. Excluíram-se as publicações duplicadas, estudos que não abordassem a temática relevante ao objetivo de revisão integrativa da literatura e que utilizassem outros métodos que não os selecionados.

O processo de identificação e seleção dos artigos decorreu em duas etapas. A primeira etapa consistiu na leitura dos títulos e resumos dos artigos identificados na pesquisa, de forma a excluir aqueles que não iam ao encontro dos critérios de inclusão previamente estabelecidos. Posteriormente, a seleção dos artigos para a continuação deste processo foi realizada com base na leitura do resumo e subtítulos 
ao longo do artigo, e na última etapa, os artigos resultantes desta primeira análise foram analisados na íntegra.

\section{Resultados}

Nas primeiras pesquisas, após a aplicação dos critérios de inclusão e exclusão, resultaram 44 artigos, tendo-se identificado 13 artigos duplicados. Realizada a leitura dos títulos e resumos, eliminaram-se sete artigos por pertencerem a periódicos de revistas que não eram considerados de cariz científico ou não respeitarem os níveis de evidência. Permaneceram 21 artigos que se dirigiam ao objetivo da revisão; no entanto, após a leitura integral, foram reduzidos a cinco publicações, por não estarem presentes os descritores considerados elegíveis à elaboração da revisão integrativa da literatura.

A Tabela 1 apresenta uma breve descrição dos objetivos e conclusões dos estudos, sendo os artigos identificados da América do Norte.

\section{Tabela 1}

\section{Síntese da Evidência Encontrada}

\begin{tabular}{|c|c|c|c|c|}
\hline Autores & Ano & País & Objetivo de Estudo & Conclusões do Estudo \\
\hline Zinan & 2014 & $\begin{array}{l}\text { Estados } \\
\text { Unidos }\end{array}$ & $\begin{array}{l}\text { Apresentar resultados de um } \\
\text { programa educacional } \\
\text { projetado para capacitar } \\
\text { enfermeiros a identificar } \\
\text { vítimas, agressores e técnicas } \\
\text { de intervenção. }\end{array}$ & $\begin{array}{l}\text { Formação direcionada aos enfermeiros } \\
\text { sobre o cyberbullying, permitiu a estes } \\
\text { profissionais de saúde uma maior } \\
\text { capacitação na identificação de sinais e } \\
\text { sintomas relacionados com o bullying e } \\
\text { identificação de vítimas e agressores. }\end{array}$ \\
\hline $\begin{array}{l}\text { Carpenter \& } \\
\text { Hubbard }\end{array}$ & 2014 & $\begin{array}{l}\text { Estados } \\
\text { Unidos }\end{array}$ & 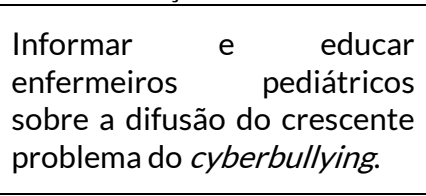 & $\begin{array}{l}\text { Enfermeiros com formação especializada } \\
\text { em saúde mental, demonstram-se mais } \\
\text { capacitados em intervir em adolescentes, } \\
\text { famílias e comunidades que experienciou } \\
\text { o cyberbullying. }\end{array}$ \\
\hline Carter \& Wilson & 2015 & $\begin{array}{l}\text { Estados } \\
\text { Unidos }\end{array}$ & $\begin{array}{l}\text { Explorar a prevalência de } \\
\text { bullying e cyberbullying em } \\
\text { adolescentes na região do } \\
\text { Midwest nos Estados Unidos. }\end{array}$ & $\begin{array}{l}\text { A prevalência do cyberbullying entre os } \\
\text { adolescentes exige a necessidade de } \\
\text { maior formação dos enfermeiros nos } \\
\text { cuidados de saúde primários na } \\
\text { identificação e intervenção precoce do } \\
\text { cyberbullying. }\end{array}$ \\
\hline $\begin{array}{l}\text { Byrne, Vessey, \& } \\
\text { Pfeifer }\end{array}$ & 2018 & $\begin{array}{l}\text { Estados } \\
\text { Unidos }\end{array}$ & $\begin{array}{l}\text { Explorar o conhecimento dos } \\
\text { enfermeiros nos cuidados de } \\
\text { saúde primários sobre o } \\
\text { cyberbullying. }\end{array}$ & $\begin{array}{l}\text { Os enfermeiros dos cuidados de saúde } \\
\text { primários revelam-se fundamentais no } \\
\text { combate ao cyberbullying, justificando-se } \\
\text { maior investimento na formação destes } \\
\text { profissionais na promoção de uma cultura } \\
\text { escolar positiva na redução de } \\
\text { comportamentos de cyberbullying. }\end{array}$ \\
\hline $\begin{array}{l}\text { Moreno, Standiford, } \\
\& \text { Cody }\end{array}$ & 2018 & $\begin{array}{l}\text { Estados } \\
\text { Unidos }\end{array}$ & $\begin{array}{lr}\text { Avaliar o papel das } \\
\text { tecnologias de informação e } \\
\text { comunicação } & \text { no } \\
\text { desenvolvimento } & \text { da } \\
\text { população adolescente. } & \end{array}$ & $\begin{array}{l}\text { Autilização das tecnologias de informação } \\
\text { e comunicação apresentam } \\
\text { consequências positivas e negativas. Os } \\
\text { enfermeiros em pediatria revelam-se } \\
\text { necessários na mobilização de estratégias } \\
\text { positivas na prevenção de } \\
\text { comportamentos do cyberbullying. }\end{array}$ \\
\hline
\end{tabular}




\section{Discussão}

As tecnologias da informação vieram reorganizar a forma como os adolescentes comunicam e aprendem, existindo a necessidade de sensibilizar não só os pais e educadores para os riscos da utilização da internet (Gil \& Félix, 2015), mas também sensibilizar os enfermeiros nos cuidados de saúde primários para esta nova realidade. Apesar de a Direção Geral de Saúde publicar uma norma do Programa Nacional de Saúde Escolar (PNSE|2015), a mesma somente faz referência à exploração indevida da utilização das TIC de uma forma menos correta, alertando para que a saúde escolar sensibilize a comunidade educativa para a importância da identificação precoce de determinadas dependências (DGS, 2015). Estudos indicam que o uso excessivo de internet pode influenciar o aparecimento de problemas emocionais e relacionais (Almeida \& Gouveia, 2016), apresentando consequências, quer a nível individual, quer a nível de saúde pública (Lopes, Patrão, \& Gouveia, 2018).

A rápida evolução das TIC possibilita de forma gratuita e sem controlo ou supervisão da forma como as crianças/jovens comunicam e constituem as suas identidades como pessoas (Pessoa \& Amado, 2014), podendo favorecer o aparecimento de distúrbios alimentares (Patchin \& Hinduja, 2006). Considera-se, assim, relevante compreender a exposição à internet (tempo despendido na internet sem ter em consideração pesquisas para trabalhos escolares), as questões sobre as preocupações com a imagem corporal (internalização dos ideais de beleza) e as questões sociodemográficas, no intuito de investigar especificamente a relação entre a exposição dos adolescentes na internete as preocupações com a imagem corporal (Tiggemann \& Miller, 2010). Apontamos, a título de exemplo, a correlação entre as preocupações com a imagem corporal e a exposição à internet, em que o Facebooke o Instagramsão consideradas as redes sociais com maior impacto (Cohen, Newton-John, \& Slater, 2017). No mesmo sentido, o estudo de Tiggemann e Slater (2013) revelou que a exposição das adolescentes à internet está associada à internalização de ideais de forma corporal, hipervigilância do corpo e à busca do ideal de magro. Estas crenças podem ser originadas pelo facto de as participantes do estudo usarem a internet pelo menos duas horas diárias na pesquisa de sites de moda, compras e revistas de celebridades, focando as preocupações com uma aparência corporal mais magra.

O bullying é considerado uma preocupação de saúde pública, caracterizado pela repetição da intimidação física ou psicológica, que através das tecnologias de informação e comunicação, e em que a intimidação psicológica inclui a humilhação, os insultos, as calunias e a deturpação da informação, sendo das características mais comuns o envio por via de email, mensagens ou blogs (Moreno \& Vaillancourt, 2017). Apesar da importância do seu reconhecimento no domínio da saúde pública, a National Association of School Nurses e a American Academy of Pediatrics revelaram que cerca de $41 \%$ dos enfermeiros mencionam que outros profissionais detêm melhor preparação para lidar com as situações de bullying, 25\% mencionaram não deter as qualificações necessárias e somente 15\% afirmaram que não havia barreiras para se envolverem em ações de prevenção do bullying (Zinan, 2014). 
A identificação da falta de formação nas escolas de enfermagem sobre o bullyinglevou à implementação de programa de intervenção intitulado "Will educating nurses about bullying empower them to become involved in preventing it and intervening?', que avaliava a eficácia de 35 métodos para reduzir o bullying, envolvendo objetivos tais como: identificar a prevalência e fatores de risco associados ao bullying, explorar atitudes e crenças relacionadas com o bullying, integrar o conhecimento sobre o bullying na sua compreensão e prevenção; explorar atitudes e crenças relacionadas com a obesidade e jovens lésbicas, gays, bissexuais e transexuais; identificar vitimas de intimidação durante a consulta de enfermagem e identificar os recursos e formas de ajudar as vítimas de intimidação. No final do programa, todos os enfermeiros evidenciaram maior capacidade de reconhecer sinais e sintomas de estudantes vítimas e agressores de bullying, identificando 14 métodos considerados mais eficazes que na redução o bullying (Zinan, 2014).

Carpenter e Hubbard (2014) mencionaram a mudança das tendências de comunicação (o tempo utilizado nas redes sociais e a maior probabilidade de o cyberbullying ocorrer); o número de histórias de cyberbullying e a emergência das tentativas ou suicídio consumado (considerado um grave problema de saúde pública); os efeitos que o cyberbullying pode ter a nível social, psicológico e académico (violência, ideação suicida, consumo de substancias, vergonha, absentismo, problemas psicossomáticos, outros); leis e punições (legislação criminal); criação de redes de suporte na avaliação e tratamento do cyberbullying (psicólogos, enfermeiros, advogados, consultores, outros). Salientaram ainda uma desconexão significativa entre o que o público necessita saber e o que realmente é conhecido sobre a gravidade da questão de saúde pública causado pelo cyberbullying e concluíram que os enfermeiros com especialidade em saúde mental estão preparados para fornecer uma série de intervenções às crianças/adolescentes que sofrem de cyberbullying (Carpenter \& Hubbard, 2014).

Recentemente, uma revisão de literatura focou principalmente nos significados e aspetos chave do cyberbullying, TIC e discrepância dos meios de utilização das TIC (essencialmente as redes sociais). Numa primeira secção abordaram as incidências do cyberbullying na sociedade e como utilizam as TIC no cyberbullying, e numa segunda secção apresentaram a emergência de três temas: divulgação, implicações na saúde e a resposta das escolas, concluindo que, quer os enfermeiros, quer as equipas multidisciplinares, podem desenvolver planos que foquem os benefícios e malefícios das redes sociais com os adolescentes (Guinta \& John, 2018). Segundo Byrne et al. (2018), um modelo de prevenção de saúde pública (primária, secundária e terciária) deve apoiar e organizar as intervenções dos enfermeiros nas escolas, defendendo os autores que a intervenção primária e secundária são mais eficazes na redução das taxas de cyberbullying. Byrne et al. (2018) defendem que os enfermeiros devem capacitar-se de maior informação sobre a prevenção do cyberbullying (e.g., conhecer bem as aplicações existentes e a terminologia utilizada), sendo o papel do enfermeiro fundamental para a promoção de uma cultura escolar positiva.

Um estudo de Pereira e Botti (2017) considerou que as TIC têm uma forte influência sobre o suicídio, tornando-se um problema de saúde pública. Estes autores concentraram-se na pesquisa de publicações 
sobre as evidências entre a comunicação através das redes sociais virtuais e o suicídio, revelando que as TIC podem apresentar dois tipos de comunicação: por um lado, a comunicação online pro-suicida, em que a internet pode operar facilitando o acesso a conteúdos sobre o suicídio, informação sobre métodos, identificação, encorajamento, entre outros e, por outro lado, a comunicação preventiva na internet apresenta-se como uma medida preventiva através de grupos de apoio online, redes sociais virtuais e apoio psicológico online. O público jovem apresenta mais vulnerabilidade na comunicação online pro-suicida pela facilidade do acesso aos meios digitais e características inerentes à adolescência, sendo assim de extrema importância aumentar a visibilidade dos sites de prevenção do suicídio (Pereira \& Botti, 2017).

Os prejuízos causados às vítimas de cyberbullying (ansiedade e depressão, doenças psicossomáticas, violência retaliatória e suicídio) têm despertado a atenção de todos os profissionais e investigadores (Sourander et al., 2010), onde a abordagem dos enfermeiros nos cuidados de saúde primários requer a familiarização do cyberbullyingna avaliação e intervenção comunitária (Carter \& Wilson, 2015). Sendo uma das competências do enfermeiro especialista em enfermagem de saúde da criança/jovem, a intervenção em situações de especial complexidade, promovendo a maximização da saúde (Regulamento n. ${ }^{\text {123/2011, }}$ 2011), e considerando que a internet é especialmente utilizada em casa da família, na qual os pais desempenham um papel fundamental na sua utilização (Maidel \& Vieira, 2015), revela-se pertinente que este profissional de saúde esteja atento e detete precocemente quando o relacionamento entre a criança/adolescente e a família/pessoa significativa é substituída pelas TIC (Moreno et al., 2018).

Apesar de os enfermeiros estarem familiarizados com o bullyingtradicional, estes têm pouco conhecimento sobre o cyberbullying (Carter \& Wilson, 2015), onde a formação especializada e direcionada a estes profissionais pode ser uma mais valia na identificação das vítimas e agressores (Carpenter \& Hubbard, 2014; Zinan, 2014). Segundo Moreno et al. (2018), as possíveis consequências das TIC no quotidiano das crianças e adolescentes, tais como as consequências educacionais (multitarefas recorrendo ao uso das TIC podem comprometer o performance académico); as consequências na saúde mental (depressão, redução da autoestima, alterações no comportamento); o sexting (mensagens eróticas, utilização de imagens seminuas ou nuas) e a privacidade (partilha de informações pessoais) (Moreno et al., 2018).

\section{Considerações finais}

O presente trabalho permite compreender a importância do papel dos enfermeiros na abordagem da problemática do cyberbullying, nomeadamente no âmbito dos cuidados de saúde primários, sendo fundamental estarem atentos e explorarem as influências menos positivas da utilização da internet no bemestar da criança/adolescente e respetivas famílias. A utilização da internet em Portugal tem vindo a sofrer aumentos consistentes, em que a grande maioria acede à internet diariamente, sendo o escalão etário entre os 15 anos que mais utiliza a internet (Cardoso, Mendonça, Lima, Paisana, \& Neves, 2014).

Estudos recentes divulgam que as redes sociais têm forte influência sobre as preocupações com a aparência nos adolescentes (de Vries, Peter, de Graaf, \& Nikken, 2016). Cohen et al. (2017) revelam uma relação entre 
as preocupações com a imagem corporal e o uso das redes sociais tais como o Facebook e Instagram. Por outro lado, os resultados de um estudo sugerem que o uso do Instagram pode influenciar negativamente as crenças e as preocupações relacionadas com a aparência (Fardouly, Willburger, \& Vartanian, 2018). Rumsey (2008) alerta os profissionais de saúde para a necessidade de uma maior atenção às implicações das preocupações com a aparência nos comportamentos relacionados com a saúde dos indivíduos (impacto negativo na perceção do self e bem-estar; intervenções cirúrgicas, adesão a tratamentos, distúrbios alimentares, etc.).

Consideramos essencial a existência de programas de apoio que dotem os enfermeiros nos cuidados de saúde primários de técnicas e métodos de avaliação e intervenção no cyberbullying, uma vez que os estudos mencionam que programas de formação especializada sobre o cyberbullying capacitam os enfermeiros a identificar vítimas e agressores (Zinan, 2014), para que os especialistas em saúde mental não sejam os únicos a possuírem essas técnicas de avaliação e intervenção (Carpenter \& Hubbard, 2014). De realçar que as equipas de enfermagem apresentam fragilidades no conhecimento sobre o comportamento suicida, influenciando assim possíveis intervenções (Reisdorfer et al., 2015), podendo esta "incapacidade" ser maior quando os enfermeiros nos cuidados de saúde primários não detêm um conhecimento profundo sobre o impacto do cyberbullying no quotidiano dos adolescentes e respetivas famílias.

Segundo Moreno et al. (2018), os enfermeiros podem desempenhar um importante papel no desenvolvimento de hábitos saudáveis na utilização das TIC por parte dos adolescentes, ficando atentos ao número de horas despendidas nas TIC; à forma como são utilizadas; aos sinais de alerta, tais como obesidade ou redução de peso, agressividade, consumo de substâncias e outros; ao número de vezes que recorrem aos cuidados de saúde; sendo importante encorajar as famílias a evitar o uso excessivo das TIC. Com base na presente revisão integrativa da literatura, enaltece-se a emergência de estudos que avaliem a capacitação dos enfermeiros em cuidados de saúde primários perante um problema de saúde pública (cyberbullying), assim como a promoção de formações especializadas para estes profissionais poderem responder eficazmente às problemáticas associadas à utilização das tecnologias de informação e comunicação (essencialmente as redes sociais virtuais) e que condicionam a saúde e bem-estar do adolescente/família.

Alertamos para o facto de existirem centenas de artigos que abordam o cyberbullying (inclusive Portugal), mas os estudos encontrados nos últimos cinco anos que façam referência à abordagem dos enfermeiros perante o cyberbullying foram somente realizados na América do Norte. Apesar de existirem diretivas que promovem a realização de estudos sobre as dependências nos cuidados de saúde (incluindo o cyberbullying) (DGS, 2015), são desconhecidos estudos realizados na Europa, sugerindo-se mais investigação perante a problemática multifacetada do cyberbullying (Moreno \& Vaillancourt, 2017). Salientamos também que atualmente existem várias redes sociais (além do Facebook) que podem influenciar a prática de cyberbullying (e.g., Instagram, Snapchat, WhatsApp, Twitter). Como limitações, consideramos o número reduzido de artigos científicos que deram resposta à questão de investigação. 
Conflito de interesses | Conflict of interest: nenhum | none.

Fontes de financiamento | Funding sources: nenhuma | none.

Contributos: JM: Revisão da literatura; redação do manuscrito. SQ: Discussão; revisão do manuscrito. MP: Discussão; revisão do manuscrito. MO: Discussão; revisão do manuscrito.

\section{Referências}

Almeida, A., \& Gouveia, P. (2016). Ciberbullying: O papel dos pares, da família e da escola [Cyberbullying: The role of peers, family and school]. In I. Patrão \& D. Sampaio (Eds.). Dependências online: O poder das tecnologias [Online addictions: The power of technology] (1st ed., pp. 75-95). Lisboa: Pactor.

Amado, J., Matos, A., Pessoa, T., \& Jäger, T. (2009). Cyberbullying: Um desafio à investigação e à formação [Cyberbullying: A challenge to research and training]. Interaç̧ões, 13, 301-326. Retrieved from https://revistas.rcaap.pt/interaccoes/article/view/409

Associação Portuguesa de Apoio à Vítima. (2011). Manual crianças e jovens vítimas de violência: Compreender, intervir e prevenir [Manual children and young victims of violence: Understanding, intervening and preventing]. Lisboa: APAV.

Betz, C. L. (2011). Cyberbullying: The virtual threat. Journal of Pediatric Nursing, 26(4), 283-284. https://doi.org/10.1016/j.pedn.2011.04.002

Byrne, E., Vessey, J. A., \& Pfeifer, L. (2018). Cyberbullying and social media: Information and interventions for school nurses working with victims, students, and families. Journal of School Nursing, 34(1), 38-50. https://doi.org/10.1177/1059840517740191

Caetano, A. P., Amado, J., Martins, M. J. D., Simão, A. M. V., Freire, I., \& Pessôa, M. T. R. (2017). Cyberbullying: Motivos da agressão na perspetiva de jovens portugueses [Cyberbullying: Motives of agression from the perspective of young portuguese]. Educação \& Sociedade, 38(141), 1017-1034. https://doi.org/10.1590/es0101-73302017139852

Cardoso, G., Mendonça, S., Lima, T., Paisana, M., \& Neves, M. (2014). A internet em Portugal - Sociedade em Rede 2014 [The internet in Portugal - Network society 2014]. Lisboa: Publicações OberCom. https://doi.org/10.13140/RG.2.1.3662.9362

Carpenter, L. M., \& Hubbard, G. B. (2014). Cyberbullying: Implications for the psychiatric nurse practitioner. Journal of Child and Adolescent Psychiatric Nursing, 273), 142-148. https://doi.org/10.1111/jcap.12079

Carter, J. M., \& Wilson, F. L. (2015). Cyberbullying: A 21st century health care phenomenon. Pediatric Nursing, 41(3), 115-125. Retrieved from https://www.pediatricnursing.net/issues/15mayjun/abstr1.html

Cohen, R., Newton-John, T., \& Slater, A. (2017). The relationship between Facebook and Instagram appearancefocused activities and body image concerns in young women. Body Image, 23, 183-187. https://doi.org/10.1016/j.bodyim.2017.10.002

Conselho da Europa. (2016). Estratégia do conselho da europa sobre os direitos da criança (2016-2021) [Strategy of the council of Europe on the rights of the child (2016-2021)]. Lisboa: Editorial do Ministério da Educação e Ciência. Retrieved from https://www.cnpdpcj.gov.pt/news/estrategia-do-conselho-da-europapara-os-direitos-da-crianca-2016-2021.aspx

de Vries, D. A., Peter, J., de Graaf, H., \& Nikken, P. (2016). Adolescents' social network site use, peer appearancerelated feedback, and body dissatisfaction: Testing a mediation model. Journal Youth Adolescence, 45(1), 211-224. https://doi.org/10.1007/s10964-015-0266-4

Direção-Geral de Saúde. (2013). Programa nacional de saúde infantil e juvenil/[National child and youth health program]. Lisboa: DGS, Ministério da Saúde. Retrieved from https://www.dgs.pt/documentos-epublicacoes/programa-tipo-de-atuacao-em-saude-infantil-e-juvenil.aspx 
Direção-Geral de Saúde. (2015). Programa nacional de saúde escolar 2015 [National school health program 2015]. Lisboa: DGS, Ministério da Saúde. Retrieved from https://observatoriolisboa.eapn.pt/ficheiro/Programa-Nacional-de-Saúde-Escolar-2015.pdf

Fardouly, J., Willburger, B. K., \& Vartanian, L. R. (2018). Instagram use and young women's body image concerns and self-objectification: Testing mediational pathways. New Media \& Society, 2044) 1380-1395. https://doi.org/10.1177/1461444817694499

Gil, H., \& Félix, C. (2015, May). Utilização segura da internet como recurso educativo na prática de ensino supervisionada [Safe use of the internet as an educational resource in supervised teaching practice]. Paper presented at the IX International Conference on ICT in education - Half a century of ICT in education, Braga, Portugal. Abstract retrieved from https://repositorio.ipcb.pt/bitstream/10400.11/2850/1/Utiliza\%C3\%A7\%C3\%A30\%20segura\%20da\%20i nternet.pdf

Guinta, M. R., \& John, R. M. (2018). Social media and adolescent health. Pediatric Nursing, 44(4), 196-201. Retrieved from https://www.pediatricnursing.net/issues/18julaug/

João, A L. S., João, B. M. S., \& Portelada, A. F. S. P. (2011). Cyberbullying: A agressão através das novas tecnologias [Cyberbullying: Agression through new technologies]. International Journal of Developmental and Educational Psychology, 2(1), 127-134. Retrieved from https://www.redalyc.org/articulo.oa?id=349832329012

Lopes, R. S., Patrão, I., \& Gouveia, M. (2018). Online time perception: Literature review. Psicologia, Saúde \& Doenças, 191), 151-156. https://doi.org/10.15309/18psd190122

Maidel, S., \& Vieira, M. L. (2015). Mediação parental do uso da internet pelas crianças [Parental mediation of internet use by children]. Psicologia em Revista, 21(2), 293-313. https://doi.org/10.5752/P.16789523.2015V21N2P292

Moreno, M. A., Standiford, M., \& Cody, P. (2018). Social media and adolescent health. Current Pediatrics Reports, 6(2), 132-138. https://doi.org/10.1007/s40124-018-0165-8

Moreno, M. A., \& Vaillancourt, T. (2017). The role of health care providers in cyberbullying. Canadian Journal of Psychiatry, 62(6), 364-367. https://doi.org/10.1177/0706743716684792

Patchin, J. W., \& Hinduja, S. (2006). Bullies move beyond the schoolyard: A preliminary look at cyberbullying. Youth Violence and Juvenile Justice, 4(2), 148-169. https://doi.org/10.1177/1541204006286288

Pereira, C., C. M. \& Botti, N. C. L. (2017). O suicídio na comunicação das redes sociais virtuais: Revisão integrativa da literatura [Suicide in the communication of virtual social networks: Integrative literature review]. Revista Portuguesa de Enfermagem de Saúde Mental, 17, 17-24. https://doi.org/10.19131/rpesm.0179

Pessoa, T., \& Amado, J. (2014). Cyberbulling - Questões e desafios atuais [Cyberbulling - Current issues and challenges]. Revista de Educación Mediática y TIC, 3(2), 29-51. Retrieved from https://helvia.uco.es/bitstream/handle/10396/13488/Edmetic_vol_3_n_2_4.pdf?sequence=1\&isAllowed=y

Pigozi, P. L., \& Machado, A. L. (2015). Bullying na adolescência: Visão panorâmica no Brasil [Bullying during adolescence in Brazil: An overview]. Ciência \& Saúde Coletiva, 2011), 3509-3522. https://doi.org/10.1590/1413-812320152011.05292014

Regulamento n. ${ }^{\circ}$ 123/2011 de 18 de fevereiro. Diário da República n. ${ }^{\circ}$ 35/2011, Série // [Regulation no. 123/2011 February 18 ${ }^{\text {th }}$. Republic Diary, no. 35/2011, $2^{\text {nd }}$ serie]. Lisboa: Ordem dos Enfermeiros. Retrieved from https://dre.pt/application/conteudo/3477012

Regulamento n. ${ }^{\circ}$ 140/2019 de 6 de fevereiro. Diário da República n. ${ }^{\circ}$ 26/2019, Série II [Regulation no. 140/2019 February $6^{\text {th }}$. Republic Diary, no. 26/2019, $2^{\text {nd }}$ serie]. Lisboa: Ordem dos Enfermeiros. Retrieved from https://dre.pt/application/conteudo/119236195

Regulamento n. ${ }^{\circ}$ 351/2015 de 22 de junho. Diário da República n. ${ }^{\circ}$ 119/2015, Série II [Regulation no. 351/2015 June 22nd. Republic Diary, no. 119/2015, $2^{\text {nd }}$ serie]. Lisboa: Ordem dos Enfermeiros. Retrieved from https://dre.pt/application/conteudo/67552235 
Reisdorfer, N., Araújo, G. M., Hildebrandt, L. M., Gewehr, T. R., Nardino, J., \& Leite, M. T. (2015). Suicídio na voz de profissionais de enfermagem e estratégias de intervenção diante do comportamento suicida [Suicide professional voice and intervention strategies before the suicidal behavior]. Revista de Enfermagem da UFSM, 5(2), 295-304. https://doi.org/10.5902/2179769216790

Rumsey, N. (2008). The psychology of appearance: Why health psychologists should "do looks". European Health Psychologist, 103), 46-50. Available from http://eprints.uwe.ac.uk/12031

Silva, J. L., Oliveira, W. A., Carlos, D. M., Lizzi, E. A. S., Rosário, R., \& Silva, M. A. I. (2018). Intervention in social skills and bullying. Revista Brasileira de Enfermagem, 71(3), 1085-1091. https://doi.org/10.1590/0034-71672017-0151

Simões, J. A. (2010). Oportunidades e riscos no uso da internet por crianças e jovens: Algumas conclusões do projecto EU Kids online [Opportunities and risks in the use of internet by children and young people: Some conclusions from the EU Kids online project]. Media \& Jornalismo, 91), 49-62. Retrieved from http://fabricadesites.fcsh.unl.pt/polocicdigital/wp-content/uploads/sites/8/2017/03/16artigo3.pdf

Sourander, A., Brunstein Klomek, A., Ikonen, M., Lindroos, J., Luntamo, T., Koskelainen, M., ... Helenius, H. (2010). Psychosocial risk factors associated with cyberbullying among adolescents: A population-based study. Archives of General Psychiatry, 677), 720-728. https://doi.org/10.1001/archgenpsychiatry.2010.79

Tiggemann, M., \& Miller, J. (2010). The internet and adolescent girls' weight satisfaction and drive for thinness. Sex Roles, 63(1-2), 79-90. https://doi.org/10.1007/s11199-010-9789-z

Tiggemann, M., \& Slater, A. (2013). NetGirls: The internet, facebook, and body image concern in adolescent girls. International Journal of Eating Disorders, 46(6), 630-633. https://doi.org/10.1002/eat.22141

United Nations Chilren's Fund. (2011). The state of the world's children 2011: Adolescence - An age of opportunity. New York, NY: UNICEF. Retrieved from https://www.unicef.org/sowc2011/pdfs/SOWC-2011Main-Report_EN_02092011.pdf

Zinan, N. (2014). Bully victim identification and intervention program for school nurses-A case study. Clinical Nursing Studies, 2(1), 45-52. https://doi.org/10.5430/cns.v2n1p45 